\title{
Course of Diabetic retinopathy following bariatric surgery in patients with Type 2 diabetes: the immediate and late impact of glycaemic control
}

Lakshminarayanan Varadhan ${ }^{1}$, Sushuma Kalidindi ${ }^{1}$, Gurmit Gill ${ }^{1}$, Hafsa Imran ${ }^{1}$, Tracy Humphreys ${ }^{2}$, George I Varughese ${ }^{1}$ and Ananth U Nayak $^{1 *}$

${ }^{1}$ Department of Diabetes and Endocrinology, University hospital North Midlands NHS Trust, Stoke on Trent, UK

${ }^{2}$ Department of Ophthalmology, University hospital North Midlands NHS Trust, Stoke on Trent, UK

\begin{abstract}
Background: Diabetic Retinopathy (DR) is a significant complication of diabetes mellitus, which can worsen during rapid fluctuations in glycaemic control. Bariatric surgery can cause sudden improvement in diabetes control which can affect DR in the short term.

Objectives: To assess the immediate and late impact of bariatric surgery on DR status in patients with type 2 diabetes mellitus and to evaluate the need for continuing retinal screening despite improvement or remission of diabetes.

Methods: A retrospective clinical observational analysis was conducted on patients who underwent bariatric surgery for type 2 diabetes. One-year pre-operative and all post-operative retinal screen images were obtained and graded using the national diabetic eye screen programme grading. Patients were categorised into three groups: No-DR, B-DR (Background DR), Ref-DR (Referable DR). Pre-operative retinal images were compared against one-year post-operative and final retinal assessment imaging.

Results: Data on 72 patients who fulfilled inclusion criteria was analysed. Mean duration of follow-up was 3.2(1-8) years. At baseline, 45 patients had No-DR (62.5\%); 24 patients had B-DR (33\%); 3 patients had Ref-DR (4.2\%). At final assessment, 43 patients had No-DR (59.7\%); 23 patients had B-DR (31.9\%); 6 patients had Ref-DR (8.3\%). Drop in $\mathrm{HbA}_{1 \mathrm{c}}$ at 1 year was a significantly higher in progressors $(29.5 \pm 12.1 \mathrm{vs} .19 .2 \pm 15.2 \mathrm{mmol} / \mathrm{mol} ; \mathrm{P}=0.038)$. On logistic regression (overall model chi-square $=37.6$ ) the $\mathrm{HbA}_{1 \mathrm{c}}$ drop at 1 year was a significant predictor for final progression in $\mathrm{DR}$ (wald=6.49; $\mathrm{B}=3.6 \pm 1.7 ; \mathrm{P}=0.040$ ) when corrected for demographics and baseline characteristics.; 6 patients progressed from No-DR to BDR at 1 year, whilst 7 progressed from No-DR to B-DR at the final assessment. Two patients progressed from B-DR to Ref-DR at 1 year, whilst 1 progressed from B-DR to Ref-DR at final assessment.
\end{abstract}

Conclusion: Our study demonstrates the variable nature of progression of DR after bariatric surgery in patients with type 2 diabetes mellitus. Long term trials are needed to identify the pattern of progression and to identify risk factors.

Abbreviations: DR: Diabetic retinopathy; T2DM: Type 2 diabetes mellitus; No-DR: No Diabetic Retinopathy; B-DR: Background Diabetic retinopathy; Ref-DR: Referable Diabetic retinopathy; IGF-1: Insulin like growth factor 1

\section{Introduction}

Bariatric surgery is being offered in increasing frequency to patients with type 2 diabetes (T2DM) to achieve weight loss and improve glucose control. Whilst patients are counselled against the common risks of bariatric surgery, its impact on diabetic retinopathy (DR) is often overlooked. According to data from the United Kingdom Prospective Diabetes Study (UKPDS), 37\% of patients with T2DM have evidence of DR at the time of diagnosis [1]. Sudden improvement of glucose control can inadvertently affect DR. Increase in IGF1(Insulin like growth factor-1) levels and retinal ischaemia have been the proposed aetiology [2-4]. Whilst patients can go into remission following bariatric surgery, the prognosis of DR remains unclear.

It has previously been published that changes in DR following bariatric surgery have been unpredictable [5]. Recently published studies, comparing routine $\mathrm{T} 2 \mathrm{DM}$ clinical care against bariatric surgery, had shown that a small but significant proportion of patients could develop sight threatening DR or maculopathy [6]. Though it is generally believed that bariatric surgery would have a favourable impact on progression of sight threatening DR, patients can get transient worsening of DR especially in the first year following surgery [6]. Three separate systematic review's and meta-analysis published on the impact of bariatric surgery on diabetic retinopathy have also concurred that available studies are not sufficient to support progression or regression of retinopathy [7-9].

The aim of our study was to assess the immediate and late impact of bariatric surgery on DR status in patients with T2DM and to evaluate the need for continuing retinal screening despite improvement or remission of diabetes.

Correspondence to: Ananth U Nayak, Consultant Physician, Department of Diabetes and Endocrinology, University hospital North Midlands NHS Trust, Stoke on Trent, ST46QG, UK, E-mail: Ananth.nayak@nhs.net

Key words: Diabetic retinopathy, Bariatric surgery, rapid glycaemic improvement Received: November 28, 2017; Accepted: Decemebr 18, 2017; Published: December 23, 2017 


\section{Methods}

A retrospective clinical observational analysis was conducted based on routine clinical care provided based on local hospital pathways and protocols, on patients who had bariatric surgery (gastric banding, laparoscopic gastric bypass surgery and vertical sleeve gastrectomy) between 2009-2014. The inclusion criteria for this analysis was (i) Patients with T2DM, (ii) who had completed at least 1 year of follow up after surgery (iii) had $\mathrm{HbA}_{1 \mathrm{c}}$ values available pre-and post-operatively (iv) retinal screening data available within a year preceding the surgery and at least one DR screening status after surgery. The data collection for follow up was stopped at March 2016 to allow at least one year of follow up to be available. All subsequent DR screening data since surgery until the study completion was included for analysis. $\mathrm{HbA}_{1 \mathrm{c}}$ done within the preceding 1 year before surgery was collected as $\mathrm{HbA}_{1 \mathrm{c}}$-Initial, $\mathrm{HbA}_{1 \mathrm{c}}$ at around one-year post operatively was labelled as $\mathrm{HbA}_{1 \mathrm{c}}-\mathrm{Y} 1$ and the $\mathrm{HbA}_{1 \mathrm{c}}$ closest to the last retinal screening date called as $\mathrm{HbA}_{1 \mathrm{c}}$-final.

DR status was derived from the National English Diabetic eye screening programme. All patients with diabetes get annual retinal screening, based on $2 \times 45$-degree retinal images from each eye after mydriasis. Images are derived and graded by trained screeners and graders. All the images for this study were double scored and graded by a tertiary trained grader based at the hospital. The retinal images are graded based on the national guidelines on grading protocols (Table 1) [10]. For the purpose of this analysis the retinal grades were grouped as: No-DR ( $\mathrm{R} 0$ in both eyes), B-DR=Background DR ( $\mathrm{R} 1$ in one or both eyes) or Ref-DR=Referable DR (R2 or R3 in one or both eyes, Maculopathy + irrespective of grade of DR).

Individual $\mathrm{HbA}_{1 \mathrm{c}}$ and change in $\mathrm{HbA}_{1 \mathrm{c}}$ from pre-op to post op were used as continuous variables in analysis. The $\mathrm{HbA}_{1 c}$ change was also used as a categorical variable ((i) $\pm 10 \mathrm{mmol} / \mathrm{mol}$ change; (ii) 10 to $20 \mathrm{mmol} / \mathrm{mol}$ change and (iii) $>30 \mathrm{mmol} / \mathrm{mol}$ change). Comparison between group means was done by ANOVA and paired t-test; the differences between frequencies/proportions by chi-square test and for statistical significance $P$ value of $<0.05$ was used. Binary logistic regression analysis was used to determine the factors predicting the progression of retinopathy. SPSS version 22 was used for analysis.

\section{Results}

Of the 509 patients referred for bariatric surgery at the end of the study period, 167 patients had had bariatric surgery. Of these, 81 patients had type 2 diabetes. Nine patients were excluded from the study based on the inclusion criteria and hence further analysis was conducted on 72 patients. $35 \%$ were men. Characteristics of our cohort who underwent bariatric surgery is shown in Table 2.

The mean duration of follow up was 3.2 years (1-8 years) and the mean $\mathrm{HbA}_{1 \mathrm{c}}$-final was 50.9 (28-131) with an average $\mathrm{HbA}_{1 \mathrm{c}}$ reduction during the period of $21 \mathrm{mmol} / \mathrm{mol}$ (5 patients had higher $\mathrm{HbA}_{1 \mathrm{c}}{ }^{-}$ final than $\mathrm{HbA}_{1 \mathrm{c}}$-initial). The percentage of $\mathrm{DR}$ at baseline and final assessment is summarized in Table 3.

\section{Initial vs. Final DR status}

At baseline before the bariatric surgery, retinopathy grades were: No-DR 45 (62.5\%); B-DR 24 (33\%), Ref-DR 3 (4.2\%) and maculopathy in $2(2.8 \%)$. Changes in retinal grades during the follow up period are summarized in the (Figure 1). Three patients had developed new maculopathy during the follow up period, all of whom had B-DR during the initial assessment. All the patients who progressed in DR status from No-DR during follow up did not progress to referable-
Table 1. National English Diabetic eye screening programme grading protocol [10].

\begin{tabular}{|c|c|c|}
\hline Retinopathy & Features & $\begin{array}{l}\text { Frequency of } \\
\text { testing }\end{array}$ \\
\hline R0 & None & Annual screen \\
\hline R1 - Background & $\begin{array}{l}\text { Microaneurysm(s) } \\
\text { Retinal haemorrhage }(\mathrm{s}) \pm \text { any exudate }\end{array}$ & $\begin{array}{l}\text { Annual screen, } \\
\text { inform diabetes } \\
\text { care team }\end{array}$ \\
\hline $\begin{array}{l}\text { R2- Pre- } \\
\text { proliferative }\end{array}$ & $\begin{array}{l}\text { Venous beading } \\
\text { Venous Loop or reduplication } \\
\text { Intraretinal microvascular abnormality (IRMA) } \\
\text { Multiple deep, round or blot haemorrhages } \\
\text { Cotton wool spots (CWS)- careful search for } \\
\text { above features }\end{array}$ & $\begin{array}{l}\text { Refer to hospital } \\
\text { eye service }\end{array}$ \\
\hline R3- Proliferative & $\begin{array}{l}\text { New vessels on disc (NVD) } \\
\text { New vessels elsewhere (NVE) } \\
\text { Pre-retinal or vitreous haemorrhage } \\
\text { Pre-retinal fibrosis } \pm \text { tractional retinal detachment }\end{array}$ & $\begin{array}{l}\text { Fast-track } \\
\text { referral to } \\
\text { hospital eye } \\
\text { service }\end{array}$ \\
\hline \multicolumn{3}{|l|}{ Maculopathy } \\
\hline M0 & Nil present & $\begin{array}{l}\text { Annual } \\
\text { screening }\end{array}$ \\
\hline M1 & $\begin{array}{l}\text { Exudate within 1disc diameter(DD) of the centre } \\
\text { of the fovea } \\
\text { Circinate or group of exudates within the macula } \\
\text { Retinal thickening within } 1 \text { DD of the centre of } \\
\text { the fovea (if stereo available) } \\
\text { Any microaneurysm or haemorrhage within 1DD } \\
\text { of the centre of the fovea only if associated with a } \\
\text { best visual acuity of } \leq 6 / 12 \text { (if no stereo) }\end{array}$ & $\begin{array}{l}\text { Refer to hospital } \\
\text { eye service }\end{array}$ \\
\hline $\begin{array}{l}\text { Photocoagulation } \\
\text { (P) }\end{array}$ & $\begin{array}{l}\text { Focal/grid to macula } \\
\text { Peripheral scatter }\end{array}$ & \\
\hline Unclassifiable (U) & Ungradeable/unobtainable & \\
\hline
\end{tabular}

Table 2. Baseline characteristics of cohort $n=72$ with type 2 diabetes who underwent bariatric surgery.

\begin{tabular}{|c|c|}
\hline Age in years (Mean \pm sd) & $49.2 \pm 8.5$ \\
\hline Gender; $\%$ Male $($ number) & $34.7 \%(25)$ \\
\hline Pre-op BMI $\left(\mathrm{kg} / \mathrm{m}^{2}\right)$ & $47.8 \pm 7.5$ \\
\hline Pre-op $\mathrm{HbA}_{1 \mathrm{c}}(\mathrm{mmol} / \mathrm{mmol})$ & $68.2 \pm 18.2$ \\
\hline Proportion with Hypertension & $73.6 \%$ \\
\hline Diabetes Treatment $(\%$ on insulin pre-op) & $23.6 \%$ \\
\hline Surgery type (LGBS vs. SG vs. GB)* & $87.5 \%$ vs. 9.7 vs. $2.8 \%$ \\
\hline
\end{tabular}

(*LGBS: Laparoscopic gastric bypass surgery; SG: Sleeve gastrectomy; GB: Gastric band)

Table 3. Percentage of DR at baseline and final assessment (Cross tabulation: $\chi^{2}=51.34$; $\mathrm{P}<0.001)$.

\begin{tabular}{|c|c|c|c|c|}
\hline \multicolumn{5}{|c|}{ DR at Final assessment } \\
\hline \multirow{4}{*}{ 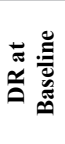 } & & No-DR & $B-D R$ & Ref-DR \\
\hline & No-DR & $36(50 \%)$ & $9(12.5 \%)$ & 0 \\
\hline & $B-D R$ & $7(9.7 \%)$ & $14(19.4 \%)$ & $3(4.2 \%)$ \\
\hline & Ref-DR & 0 & 0 & $3(4.2 \%)$ \\
\hline
\end{tabular}

DR. Patients who had Ref-DR at initial assessment remained at the same status throughout the study. Of the entire cohort 9 patients had progression of DR during the period of follow up and were classed as 'progressors' for further analysis ( 9 progressed from R0 to B-DR; 3 progressed from B-DR to Ref-DR).

A comparison between progressors and stable patients (including patients whose DR grades improved) is shown in Table 4. This showed that the drop in $\mathrm{HbA}_{1 \mathrm{c}}$ at 1 year was a significantly higher in progressors $(29.5 \pm 12.1$ vs. $19.2 \pm 15.2 \mathrm{mmol} / \mathrm{mol}$; $\mathrm{P}=0.038)$. On logistic regression (overall model chi-square $=37.6$ ) the $\mathrm{HbA}_{1 \mathrm{c}}$ drop at 1 year was a significant predictor for final progression (wald=6.49 B=3.6 $\pm 1.7 ; \mathrm{P}=0.040$ ); Age, $\mathrm{BMI}$ at baseline, presence of hypertension pre-op, and baseline $\mathrm{HbA}_{1 \mathrm{c}}$ were all not significant. 


\section{Initial vs. Year 1(Y1) vs Final DR status}

The details of DR status change comparing initial assessment to first year of follow up to final DR are shown in (Figure 2). Four patients from the No-DR group and 1 from the B-DR group did not have the DR screening at Y1 but had the DR screening subsequently (hence included in the final DR analysis). A number of patients tend to progress or improve their retinal status with time. During follow up, 6 patients progressed from No-DR to B-DR at Y1; 5 of these 6 reverted back to No-DR at final assessment. Of the 4 from B-DR group that improved to no-DR at $\mathrm{Y} 1,3$ continued as no-DR but 1 reverted back to $\mathrm{B}-\mathrm{DR}$ at final assessment.

\section{Discussion}

The data from this study again shows that patients with T2DM who undergo bariatric surgery, have variable progression of DR during

Table 4. Comparing Progressors in DR vs. those with Stable DR.

\begin{tabular}{|c|c|c|c|}
\hline & $\begin{array}{c}\text { Progressors } \\
(\mathbf{n}=\mathbf{1 2})\end{array}$ & $\begin{array}{c}\text { Stable patients } \\
(\mathbf{n}=\mathbf{6 0})\end{array}$ & \\
\hline Age (years) & $49.6 \pm 10.0$ & $49.1 \pm 8.2$ & $\mathrm{P}=0.85$ \\
\hline Insulin treated (\%) & $33.3 \%$ & $20 \%$ & $\mathrm{P}=0.51$ \\
\hline Pre OP BMI (kg/m2) & $47.3 \pm 5.7$ & $47.9 \pm 7.9$ & $\mathrm{P}=0.79$ \\
\hline $\mathrm{HbA}_{1 \mathrm{c}}-$ Initial $(\mathrm{mmol} / \mathrm{mol})$ & $74.7 \pm 19.1$ & $66.7 \pm 17.9$ & $\mathrm{P}=0.156$ \\
\hline $\mathrm{HbA}_{1 \mathrm{c}}-$ at year $1(\mathrm{Y} 1)$ & $47.3 \pm 14.2$ & $47.3 \pm 13.4$ & $\mathrm{P}=0.99$ \\
\hline $\mathrm{HbA}_{1 \mathrm{c}}$ reduction-Y1 & $29.5 \pm 12.1$ & $19.2 \pm 15.2$ & $\mathrm{P}=0.038$ \\
\hline $\mathrm{HbA}_{1 \mathrm{c}}$ final & $57.9 \pm 22.4$ & $49.3 \pm 18.9$ & $\mathrm{P}=0.147$ \\
\hline $\mathrm{HbA}_{1 \mathrm{lc}}$ reduction-final & $20.9 \pm 15.4$ & $18.2 \pm 8.0$ & $\mathrm{P}=0.613$ \\
\hline
\end{tabular}

Baseline

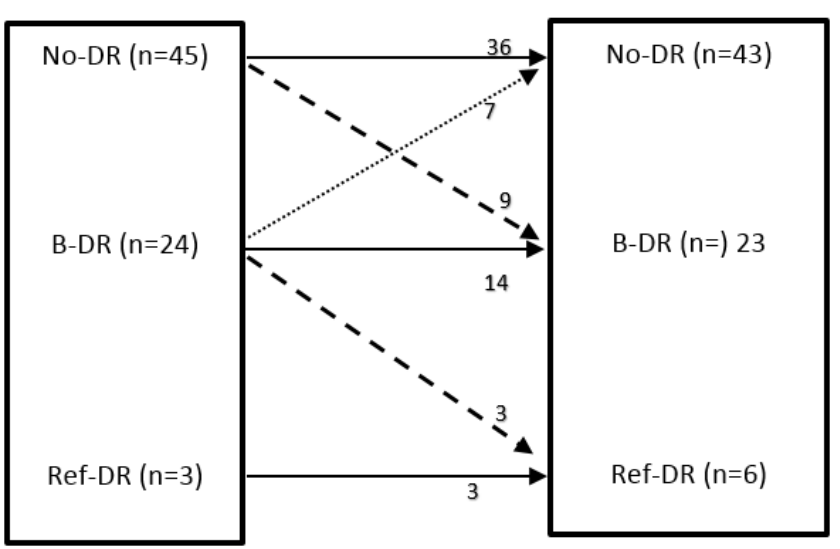

Figure 1. Changes in retinal grades following bariatric surgery: Baseline vs. final assessment post bariatric surgery. (No-DR= No Diabetic retinopathy, B-DR= Background diabetic retinopathy, Ref-DR= Referable diabetic retinopathy)

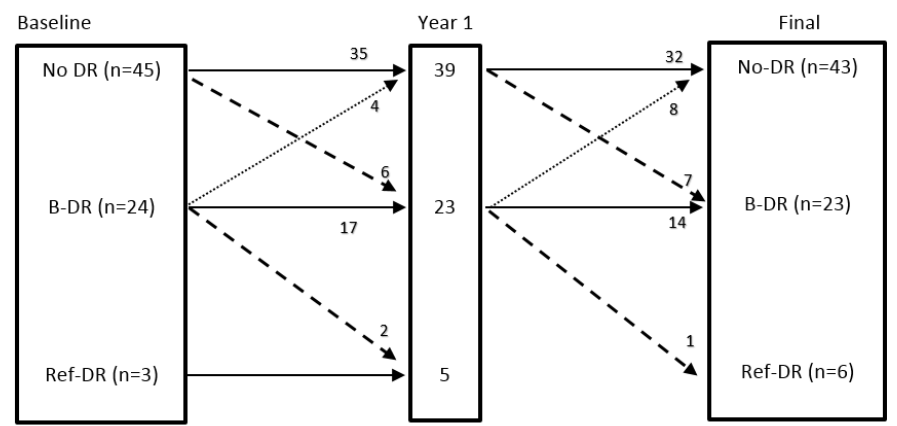

Figure 2. Changes in the retinal grades following bariatric surgery: Baseline vs. Year 1 vs. final retinal assessment (final includes data on individuals who did not have DR screening at Y1). follow up period, with dynamic changes evolving with time, and hence reiterating the importance of continuing retinal screening in this cohort.

Bariatric surgery is evolving as an important treatment option for patients with T2DM. Numerous studies have demonstrated in the past that improvement in diabetes and metabolic parameters such as hypertension and dyslipidaemia with bariatric surgery is associated with significant improvement of microvascular complications and cardiovascular risk [1,11]. The Swedish obesity study (SOS) demonstrated bariatric surgery reduced the incidence of myocardial infarction (29\%), stroke (34\%) and overall mortality (30-40\%) [12]. It has also been well established that diabetes remission could be achieved with significant proportion of these patients [13]. Remission rates of diabetes vary greatly and even more so depending on which criteria is used to consider remission. Some studies have quoted a remission rate of $43-92 \%$ [14].

It is well known that rapid improvement of $\mathrm{HbA}_{1 \mathrm{c}}$ can be associated with worsening of microvascular complications; this has been established with insulin initiation, insulin pump therapy and GLP-1 agonist therapy [15-19]. Bariatric surgery is yet another intervention that could achieve substantial improvement in glycaemic control in relatively shorter duration but could worsen DR in the immediate phase [6]. However, in the longer term, it is well known that improved glycaemic control would have significant benefits in improving DR [1]. Further concurrent improvement achieved in metabolic parameters such as hypertension and lipid profile would also contribute to this improvement and protection $[1,11]$. On the contrary, short term benefits in DR may be masked if metabolic memory is playing a role [20]. Previous studies have demonstrated these variable progressions of DR after bariatric surgery in short and long term [5-6]. Our study again demonstrates the variable nature of progression of DR following bariatric surgery and the difficulties in predicting the direction of progression of DR after bariatric surgery.

Two separate systematic review and meta-analysis were performed in 2015/2016, one in Canada and the other in Korea. The Canadian group reviewed 4 out of a total 227 studies ( $n=148$ patients). Whilst they noted that patients with a pre-existing diagnosis of DR are at increased risk following surgery, it was felt further studies are needed to evaluate this in detail [7]. The Korean group reviewed twelve studies composed of 876 patients with T2DM who underwent bariatric surgery. This review noted that remission of diabetes couldn't prevent the development or progression of DR after surgery [8]. A further systematic review and meta-analysis of controlled clinical studies was done by an Italian group to compare the rate of appearance and progression/regression of DR in studies comparing medical and surgical treatment of T2DM [9]. The authors in this case concluded that bariatric surgery seems to prevent new cases of diabetic retinopathy but acknowledged available studies are not sufficient to support progression or regression of retinopathy [9].

In our study, majority of patients with no-DR (35/45, 78\%) maintained this status up to Y1 following which $7 / 35$ then progressed to B-DR. This could be in keeping with the duration of diabetes increasing during the follow up period or attributed to other metabolic factors, data regarding which were not included in this study. However, overall 31/45 patients had maintained as no-DR throughout the study (69\%). Patients with B-DR at baseline progress even more differently with $17 / 24$ continuing to stay as BDR at $\mathrm{Y} 1 ; 13 / 24$ of these continuing as BDR till final assessment (54\%) and 1 progressing to referable- 
DR. Maintenance of B-DR and no-DR status in majority of patients throughout the study may be attributable to the long-term benefits derived with weight loss, glycaemic control and improvement of metabolic factors with bariatric surgery. A systemic review and metaanalysis done by the Canadian group Cheung et al. [7] noted that patient's with diagnosis of DR prior to surgery are at increased risk of further progression in their disease. Tahrani et al. [6] compared patients who underwent bariatric surgery against patients who had routine care. A small but significant proportion of their patients who underwent bariatric surgery developed sight threatening DR, even if they didn't have any evidence before surgery.

The phenomenon of rapid progression of DR transiently following rapid glycaemic change and subsequent improvement in DR with time is well documented before. In our study, at Y1 follow up, 6 patients from no-DR had progressed to B-DR; 5 patients reverted back to noDR by final assessment. The number of people who progress to sight threatening retinopathy or referable DR or maculopathy is small. This is again comparable to previous published studies [6]. None of the patients with No-DR at initial assessment progressed to referable-DR during the follow up period. The 2 patients who progressed to referable$\mathrm{DR}$, had antecedent B-DR and progressed within the Y1, which could a pointer towards rapid improvement of diabetes control. Based on the above two findings, it is therefore vital that patients definitely get their retinal screening continued, especially in the first year after bariatric surgery, to document these progressive changes. It is even more pertinent, when the term 'cure' for diabetes is used interchangeably with 'remission' for diabetes and patients are being removed from retinal screening programmes.

It is very difficult to establish predictors from progressive DR on a retrospective analysis. Table 4 shows the various factors that we compared, dividing the group as progressors and stable patients. Previous publications on GLP-1 agonist therapy and DR had established that duration of diabetes, $\mathrm{HbA}_{1 \mathrm{c}}$ at baseline and rapidity of reduction to be possible risk factors [21]. The degree of $\mathrm{HbA}_{1 \mathrm{c}}$ reduction achieved during Y1 after surgery was statistically significant predictor for progression of DR at the final screening, when corrected for demographics and baseline characteristics. Patients with previous known B-DR obviously appear to have the risk of progression to referable-DR. whilst this didn't reach statistical significance in our study, it is likely a marker of higher risk and may therefore help in targeting DR screening during follow up.

Dramatic improvement in weight loss and glycaemic control following bariatric surgery is most often noted within the first year. Patients are routinely counselled on the risks of anaesthetic, bleeding, infection, failure and anastomotic leak following bariatric surgery. Our study shows that 6 out of 45 patients with No-DR progressed to B-DR at one year and 2 out of 24 patients from B-DR to Ref-DR. Given the recent emerging evidence from our study as well as published literature, patients should be informed of the risk of progression of DR especially in the first year following surgery. Some studies have shown that transient worsening of DR status can occur within the first year following bariatric surgery [6]. Though the numbers are small, given no obvious predictive factors, it may be advisable to recommend an early interim retinal assessment (eg- at 4 months) post operatively especially for the B-DR patients, to identify these patients early and arrange appropriate ophthalmology input. This protocol could be very comparable to patients who go through pregnancy with diagnosis of T2DM, where similar phenomenon could happen.
Informing patients of the potential deterioration with regards to the DR might empower then to seek medical opinion if they do observe any visual changes. This might also improve patient compliance especially with regards to attending screening appointments, especially if the patients have come off all anti-diabetic medication too. The Y1 DR screening is also crucial to identify these patients and refer appropriately to retinal tertiary services. This may be even more pertinent in places such as England, where retinal screening is managed independently from the health care service. This dichotomy of service provision therefore calls for close liaison and multi-disciplinary working between endocrinologist, bariatric surgeons and retinal screening service to devise pathways for optimizing follow up for these patients.

There are obvious limitations to this study: (1) Our study is based on retrospective observational analysis, based on routine clinical practice with no discrete research intervention. Hence in some instances the duration between $\mathrm{HbA}_{1 \mathrm{c}}$ check and DR screening could not be matched. (2) As the retinal screening programme runs as an independent system in England, we do not have control on periodicity of screening or details on non-attendances and hence data on some patients during the interim period of follow up could be missing or lost. (3) The number of patients in each of the groups is small and hence the conclusions derived need to be interpreted with caution and validated in larger prospective randomized trials, to facilitate formalizing guidelines for management. (4) Data on weight, BMI and other medical conditions such as hypertension at the final visit was not available which could be a confounding factor to the incidence and prevalence of DR.

\section{Conclusion}

In conclusion, our study demonstrates the variable nature of progression of DR after bariatric surgery in patients with T2DM. It is very vital that patients continue with retinal screening programme despite improvement or remission in diabetes, especially if they already have established background retinopathy. A small number of patients could progress to referable DR or maculopathy. Transient worsening of DR could happen during the first year of follow-up with variable direction of progression during follow up period. Majority of patients might maintain their retinal status during the follow up period which could be the clinical marker of metabolic protection offered by bariatric surgery. Long term randomized controlled trials are required to identify the pattern of progression and risk factors that would help to rationalize screening and treatment for this special cohort of patients.

\section{Author Contributions}

LV, GG, HI, TH collected the data. TH has directly contributed to the grading of the digital images as well as quality assurance assessment. LV developed the research idea, analysed the results, and wrote the manuscript with AUN and GIV. SK contributed to the manuscript.

\section{References}

1. Stratton IM, Kohner EM, Aldington SJ, Turner RC, Holman RR, et al. (2001) UKPDS 50: risk factors for incidence and progression of retinopathy in Type II diabetes over 6 years from diagnosis. Diabetologia 44: 156-163. [Crossref]

2. Chantelau E (1998) Evidence that upregulation of serum IGF-1 concentration can trigger acceleration of diabetic retinopathy. Br J Ophthalmol 82: 725-730. [Crossref]

3. Grunwald JE, Riva CE, Martin DB, Quint AR, Epstein PA (1987) Effect of an insulin-induced decrease in blood glucose on the human diabetic retinal circulation. Opthalmology 94: 1614-1640. [Crossref]

4. Henricsson M, Berntorp K, Fernlund P, Sundkvist G (2002) Progression of retinopathy in insulin-treated type 2 diabetic patients. Diabetes Care 25: 381-385. [Crossref]

5. Varadhan L, Humphreys T, Walker A, Cheruvu C, Varughese G (2012) Bariatric Surgery and Diabetic Retinopathy: a Pilot Analysis. Obes Surg 22: 515-516. [Crossref] 
6. Amin AM, Wharton H, Clarke M, Syed A, Dodson P, et al. (2016) The impact of bariatric surgery on retinopathy in patients with type 2 diabetes: a retrospective cohort study. Surg Obes Relat Dis 12: 606-612. [Crossref]

7. Cheung D, Switzer NJ, Ehmann D, Rudnisky C, Shi X, et al. (2015) The Impact of Bariatric Surgery on Diabetic Retinopathy: A Systematic Review and Meta-Analysis. Obes Surg 25: 1604-1609. [Crossref]

8. Kim Y, Kim B, Choi B, Sun H, Lee S, et al. (2017) Bariatric surgery is associated with less progression of diabetic retinopathy: A systematic review and meta-analysis. Surg Obes Relat Dis 13: 352-360. [Crossref]

9. Merlotti C, Ceriani V, Morabito A, Pontiroli AE (2017) Bariatric surgery and diabetic retinopathy: a systematic review and meta-analysis of controlled clinical studies. Obes Rev 18: 309-316. [Crossref]

10. Harding S, Greenwood R, Aldington S, Gibson J, Owens D, et al. (2003) Grading and disease management in national screening for diabetic retinopathy in England and Wales. Diabet Med 20: 965-971. [Crossref]

11. Barrett A, Bakhshi S, Nayak A, Varughese G, Cheruvu C, et al. (2014) Metabolic improvement after bariatric surgery: an overview from a single tertiary centre. Endocrine Abstracts 34: 212.

12. Sjöström L (2013) Review of the key results from the Swedish Obese Subjects (SOS) trial - a prospective controlled intervention study of bariatric surgery. $J$ Intern Med 273: 219-234.

13. Ardestani A, Rhoads D, Tavakkoli A (2015) Insulin Cessation and Diabetes Remission After Bariatric Surgery in Insulin-Treated Type 2 Diabetic Adults. Diabetes Care 38: 659-664. [Crossref]
14. Mas-Lorenzo, A., Benaiges, D., Flores-Le-Roux, J., Pedro-Botet, J., Ramon, J., Parri, A., Villatoro, M., Chillarón, J., Pera, M., Grande, L. and Goday, A. (2014). Impact of Different Criteria on Type 2 Diabetes Remission Rate after Bariatric Surgery. Obes Surg 24: 1881-1887. [Crossref]

15. Vestgaard M, Ringholm L, Laugesen CS, Rasmussen KL, Damm P, et al. (2009) Pregnancy-induced sight-threatening diabetic retinopathy in women with type 1 diabetes. Diabet Med 27: 431-435. [Crossref]

16. Dahl-Jorgensen, K., Brinchmann-Hansen, O., Hanssen, K., Sandvik, L. and Aagenaes, O. (1985). Rapid tightening of blood glucose control leads to transient deterioration of retinopathy in insulin dependent diabetes mellitus: the Oslo study. Br Med J (Clin Res Ed) 290: 811-815. [Crossref]

17. Rasmussen KL, Laugesen CS, Ringholm L, Vestgaard M, Damm P, et al. (2010) Progression of diabetic retinopathy during pregnancy in women with type 2 diabetes. Diabetologia 53: 1076-1083. [Crossref]

18. Henricsson M, Nilsson A, Janzon L, Groop L (1997) The Effect of Glycaemic Control and the Introduction of Insulin Therapy on Retinopathy in Non-insulin-dependent Diabetes Mellitus. Diabet Med 14: 123-131. [Crossref]

19. Ballegooie E, Hooymans J, Timmerman Z, Reitsma W, Sluiter W, et al. (1984) Rapid Deterioration of Diabetic Retinopathy during Treatment with Continuous Subcutaneous Insulin Infusion. Diabetes Care 7: 236-242. [Crossref]

20. Reddy MA, Zhang E, Natarajan R (2015) Epigenetic mechanisms in diabetic complications and metabolic memory. Diabetologia 58: 443-455. [Crossref]

21. Varadhan L, Humphreys T, Hariman C, Walker A, Varughese G (2011) GLP-1 agonist treatment: Implications for diabetic retinopathy screening. Diabetes Res Clin Pract 94: e68-e71. [Crossref]

Copyright: (C2017 Varadhan L. This is an open-access article distributed under the terms of the Creative Commons Attribution License, which permits unrestricted use, distribution, and reproduction in any medium, provided the original author and source are credited. 